\title{
Molecular organization of the mucins and glycocalyx underlying mucus transport over mucosal surfaces of the airways
}

\author{
M Kesimer ${ }^{1,2}$, C Ehre ${ }^{1}$, KA Burns $^{1}$, CW Davis ${ }^{1,3}$, JK Sheehan ${ }^{1,2}$ and RJ Pickles ${ }^{1,4}$
}

Mucus, with its burden of inspired particulates and pathogens, is cleared from mucosal surfaces of the airways by cilia beating within the periciliary layer (PCL). The PCL is held to be "watery" and free of mucus by thixotropic-like forces arising from beating cilia. With radii of gyration $\sim 250 \mathrm{~nm}$, however, polymeric mucins should reptate readily into the PCL, so we assessed the glycocalyx for barrier functions. The PCL stained negative for MUC5AC and MUC5B, but it was positive for keratan sulfate (KS), a glycosaminoglycan commonly associated with glycoconjugates. Shotgun proteomics showed KS-rich fractions from mucus containing abundant tethered mucins, MUC1, MUC4, and MUC16, but no proteoglycans. Immuno-histology by light and electron microscopy localized MUC1 to microvilli, MUC4 and MUC20 to cilia, and MUC16 to goblet cells. Electron and atomic force microscopy revealed molecular lengths of 190-1,500 nm for tethered mucins, and a finely textured glycocalyx matrix filling interciliary spaces.

Adenoviral particles were excluded from glycocalyx of the microvilli, whereas the smaller adenoassociated virus penetrated, but were trapped within. Hence, tethered mucins organized as a space-filling glycocalyx function as a selective barrier for the PCL, broadening their role in innate lung defense and offering new molecular targets for conventional and gene therapies.

\section{INTRODUCTION}

The conducting airways of the lung provide mechanical, chemical, and immunological barriers to inhaled particulates, including respiratory pathogens. One of the first lines of defense for the lung is the mucus layer lining the tracheobronchial airways. The appropriate production and effective clearance of mucus is essential for respiratory sterility and health. The critical function of mucus clearance is highlighted by lung diseases in which mucus clearance is reduced, i.e., cystic fibrosis, the chronic bronchitis component of chronic obstructive pulmonary disease, and asthma. Effective clearance occurs by the unidirectional transport of mucus over an underlying bed of beating cilia, or, secondarily, by cough clearance. The effective transport of a soluble, viscoelastic mucus layer is dependent not only on the ciliary beat, but also on the maintenance of the fluid and ion transport mechanisms required for homeostasis within the airway surface microenvironment. ${ }^{1,2}$

Our current understanding of mucociliary clearance in the airways dates to important observations in the 1930s by Florey et al. ${ }^{3}$ who described the sources and transport of mucus in cat tracheas, and by Lucas and Douglas ${ }^{4}$ who recognized the clear separation between the mucus layer and the ciliary activity underlying its transport over the nasal epithelia of several mammals. More recently, histological preparations of airway epithelia fixed to preserve the mucus layer showed two distinctive gel-like layers, the soluble transporting mucus layer and the periciliary layer $\left(\mathrm{PCL} ;{ }^{5,6}\right)$. The soluble mucus layer is rich in the two major polymeric mucins, MUC5AC and MUC5B, and hundreds of globular proteins. ${ }^{7-10}$ Estimates of the mucus layer thickness are variable, depending on choice of fixatives and methods of measurement. Electron microscopy (EM) studies in different species measured the mucus layer as $7-10 \mu \mathrm{m}$ thick, ${ }^{5}$ whereas others report the layer to be $0.5-70 \mu \mathrm{m}$ in thickness. ${ }^{6,11,12}$

The thickness or depth of the PCL has been easier to determine as the PCL extends over the length of fully extended cilia. Dependent on airway region, cilia are between 5 and $8 \mu \mathrm{m}$ in

\footnotetext{
${ }^{1}$ Cystic Fibrosis/Pulmonary Research and Treatment Center, University of North Carolina, Chapel Hill, North Carolina, USA. ${ }^{2}$ Department of Biochemistry and Biophysics, University of North Carolina, Chapel Hill, North Carolina, USA. ${ }^{3}$ Department of Cell and Molecular Physiology, University of North Carolina, Chapel Hill, North Carolina, USA. ${ }^{4}$ Department of Microbiology and Immunology, University of North Carolina, Chapel Hill, North Carolina, USA. Correspondence: M Kesimer (mehmet_kesimer@ med.unc.edu) 


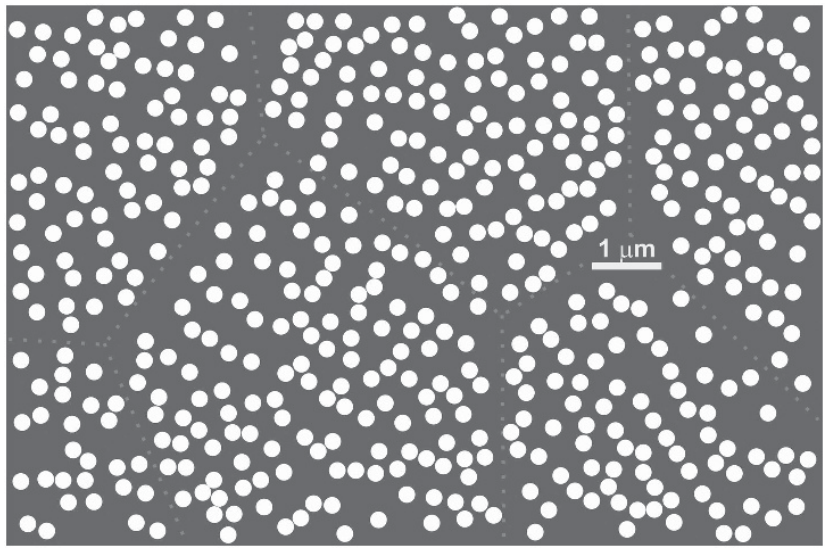

Figure 1 Density map of cilia on airway epithelial cells. Using a published electron micrograph (Figure 3 in ref. 60) of guinea-pig tracheal epithelial cells, individual cilia were mapped, manually, and are displayed as white circles. Reverse contrast was used to emphasize interciliary spaces. The micrograph represented a cross-sectional plane through the basal region of the cilia, where microvilli were apparent between the cilia, so the distribution approximates the positions of the basal bodies. Red dotted lines indicate approximate cell borders. Note the magnitude of interciliary distances, $\sim 100 \mathrm{~nm}$ to $>1 \mu \mathrm{m}$, especially between cilia on adjacent cells. A full colour version of this figure is available at the Mucosal Immunology journal online.

length. The microenvironment of the PCL surrounding the cilia has been described as "watery", or low-viscosity, to enable cilia to beat unimpeded. ${ }^{4,5,13-16}$ For this reason, the PCL is often termed the periciliary liquid layer.

A major biophysical question is why the soluble mucus layer and the PCL are maintained as a two-layer system. Earlier work suggested the intrusion of mucus into the PCL was prevented by thixotropic-like forces provided by the beating cilia. $4,13,16$ However, given the $\sim 250 \mathrm{~nm}$ radius of gyration of polymeric mucins $s^{17,18}$ and the density of cilia on a ciliated airway epithelial cell, it is unlikely that such molecules can be excluded from interciliary spaces, which approach $\mu \mathrm{m}$ distances, particularly at intercellular borders (Figure 1).

We now provide evidence for a glycocalyx more robust than previously realized (see ref. 19) that results from a complex organization of membrane-tethered, high MW glycoconjugates. Previously, we have shown that tethered mucins in the airway glycocalyx can restrict virus penetration into the airway epithelial surface. ${ }^{20,21}$ This finding, plus the recent observation in vascular endothelium that tissue preparation appropriate to the preservation of glycocalyx revealed it to be $\sim 10 \mu \mathrm{m}$ in thickness, rather than the negligible thickness observed by conventional light and electron microscopies, ${ }^{22}$ make a glycocalyx barrier to mucus penetration of the PCL a particularly attractive hypothesis.

\section{RESULTS}

\section{Spatial distribution of major mucins and} glycosaminoglycans in mucus

The major gel-forming or polymeric mucins of airway mucus have been identified as MUC5AC and MUC5B. ${ }^{23,24}$ Although airway mucus secretions normally undergo unidirectional transport in the airways in vivo, in human tracheobronchial epithelial (HTBE) cultures mucus secretions are transported within the culture dish but are not cleared. This property of HTBE cell cultures was exploited to visualize the spatial distribution of the mucins in mucus allowed to accumulate on culture surfaces over several days. As formalin-based fixatives can collapse mucus structures, we opted to use an alcohol-based fixative (Omnifix) to better preserve the mucus secretions intact during histological processing and analysis. ${ }^{25}$ Probing histological sections of HTBE with MUC5AC monoclonal and MUC5B polyclonal antibodies (see Table 1) revealed the presence of these mucins in extensive "clouds" emanating from the epithelium but with minimal spatial mingling (Figure 2c). Notably, immunodetectable MUC5AC and MUC5B were absent from a broad region immediately above the HTBE cell surface, which corresponded to the airway surface region occupied by cilia (Figure 2a,b). Although the mucosal surface of the epithelium is irregular (Figure 2a), the MUC5AC and MUC5B exclusion zone was generally $\sim 7 \mu \mathrm{m}$ high, consistent with the height of human tracheobronchial cilia. We propose that this MUC5AC/B exclusion zone represents the PCL.

Glycosaminoglycans are unbranched polysaccharide chains species normally associated with glycoconjugates of connective tissue and glycocalyx. Heparan sulfate and keratan sulfate (KS) glycosaminoglycans are classically associated with proteoglycans in connective tissue, but also associate with mucins in mucus of the airways and elsewhere. ${ }^{26-28}$ Immunoprobing histological sections of HTBE and accumulated mucus secretions failed to detect heparan sulfate at the mucosal surface of HTBE cells, either in association with the apical surface or mucus secretions (data not shown). Instead, heparan sulfate was immunodetected in the basal cell layers of the HTBE cultures as described previously. ${ }^{29}$ By contrast, KS was detected robustly on the apical surfaces of HTBE and in the mucus secretions (Figure 2d). Notably, however, the spatial distribution of KS was markedly different to that of MUC5AC and MUC5B: the most intense KS staining was within the PCL, rather than in the mucus layer, and it was particularly associated with the apical surfaces of ciliated cells. This observation suggested that KS more closely associated with glycoconjugates in close proximity to the cilia or ciliated cell apical membranes. The mucosal surfaces of ciliated cells have previously been associated with an acidic mucosubstance, ${ }^{30}$ which we now propose is KS, at least in part. Notably cilia also stained positive with alcian blue/periodic acid-Schiff (Figure 2b), as well as with the KS-specific antibody (see also Supplementary Figure S1 A online). Interestingly, many of the most intensely stained, KSrich areas had irregular interfaces with the overlying mucus layers (arrows, Figure 2d), which suggested the attachment of KS-associated material to cilia, and/or its transfer from ciliated zones of the PCL into the mucus. Notably, these "ciliary plumes", are also present in the other sections of Figure 2, as indicated by labeled arrows, whether by positive staining (Figure 2a,b,d), or by its absence (Figure 2c). Significantly, the 
Table 1 Summary of antibodies used for immunodetection

\begin{tabular}{|c|c|c|c|}
\hline Target & Antibody/staining & Epitope/region & Ref. \\
\hline MUC1 & 214D4 (mouse lgG1) & Recognizes unknown epitope at the glycosylated repeat region & Ref.63 \\
\hline MUC4 & IG8 (mouse lgG1) & Recognizes intracellular immature molecule & (this paper) \\
\hline MUC5AC & 45M1 (mouse IgG1) & C-terminal cystein-rich part & Ref.65 \\
\hline \multirow[t]{2}{*}{ MUC5B } & EU-MUC5Ba (mouse IgG1) & $\begin{array}{l}\text { Recognizes the peptide (RNREQVGKFKMC) located in repeated } \\
\text { cysteine-rich domain }\end{array}$ & Ref.66 \\
\hline & MAN5B/LUM5B (rabbit) & $\begin{array}{l}\text { Recognizes peptide (EIGQVVECSLDFGLVCR) located in the } \\
\text { cysteine repeat }\end{array}$ & Ref.8 \\
\hline MUC20 & MUCH2O (rabbit) & Recognizes C-terminal peptide (LSVASPEDLTDPR) & $\begin{array}{l}\text { Ref.35 and } \\
\text { (this paper) }\end{array}$ \\
\hline Keratan sulphate & 5D4 (mouse IgG1) & $\begin{array}{l}\text { Recognizes } N \text {-acetyl lactosamine disaccharides of KS with } \\
\text { a pentasulfated hexasaccharide. }\end{array}$ & Ref.67 \\
\hline Carbohydrate & AB/PAS (alcian blue/PAS) & $\begin{array}{l}\text { AB stains acidic (sulphated) mucins and proteoglycans, PAS stains } \\
\text { mucins and, glycoproteins, primarily their terminal sialic acid } \\
\text { moieties, but also other carbohydrates, including glycogen. }\end{array}$ & \\
\hline
\end{tabular}

Abbreviations: KS, keratan sulfate; PAS, periodic acid-Schiff.

"negative staining" of with MUC5AC and MUC5B antibodies in these areas indicate their exclusion from within the ciliary plumes.

\section{Molecular identification of the KS-associated conjugate in HTBE cell culture secretions}

To identify the molecular species associated with KS immunoreactivity, we isolated KS-rich material from HTBE cell culture washings solubilized in $4 \mathrm{M} \mathrm{GuHCl}$ and separated the material by isopycnic density gradient centrifugation in $\mathrm{CsCl} / 4 \mathrm{M}$ $\mathrm{GuHCl}^{31,32}$ The gradient was fractionated and the fractions analyzed for density, MUC5AC, MUC5B, and KS immunoreactivity, and periodic acid-Schiff staining. An agarose western blot probed with an antibody against MUC5B (Figure 3a, top) shows that the polymeric mucin resolved in the middle of the density gradient $\left(\right.$ densities $\left.=1.30-1.35 \mathrm{~g} \mathrm{ml}^{-1}\right)$. MUC5AC had a similar gradient distribution (data not shown). By contrast, KS-positive materials resolved in fractions with the highest densities (Figure 3a, bottom, densities $=1.45-1.55 \mathrm{~g} \mathrm{ml}^{-1}$ ), and with minimal apparent overlap with the MUC5AC/MUC5Brich fractions.

Similar results were also obtained using an alternative separation of HTBE cell culture secretions using a MonoQ 5/5 ion exchange column eluted over a range of salt strengths, $0.1-1.0 \mathrm{M}$ $\mathrm{LiClO}_{4}$ (Figure 3b). Fraction subsamples were blotted and probed with antibodies specific to MUC5B, MUC5AC, MUC1, MUC4, MUC16, and KS. The KS probe bound to molecules that eluted in a range generally more acidic than MUC5B: the overlap between the two elution profiles, though significant, was small and the peak responses were well separated. On the other hand, the distribution of MUC16 overlapped broadly with that of both MUC5B and KS. The distribution of MUC5AC was very similar to that for MUC5B (not shown). MUC1 had a bipolar distribution, one population of molecules more-or-less co-eluted with MUC5B, the other with MUC16. A smaller amount of MUC4 eluted from the column, with a peak positioned between those for MUC5B and MUC16. Hence, for fractions eluting at acidic pHs there was significant overlap between the presence of the tethered mucins (MUC 1, 4, and 16) and KS-conjugated molecules. Notably, approximately half of the KS-positive material eluted in fractions more acidic than those containing tethered mucins (Figure 3b), suggesting the presence of other, unidentified glycoconjugates, glycolipids, or other materials.

A two-dimensional shotgun proteomics-based approach was used to identify the proteins conjugated to KS. The first dimension was either the $\mathrm{CsCl}$ density gradient centrifugation or ion exchange chromatography separation described above (Figure 3). The KS-rich fractions (15-20) from each separation were combined, reduced, alkylated, digested with trypsin, and analyzed by ultra-performance liquid chromatography/mass spectrometry as described previously. ${ }^{7}$ MUC16 and MUC5B were identified as the predominate proteins in the KS-rich material of both separations (Table 2), using the total number of tryptic peptides identified per 

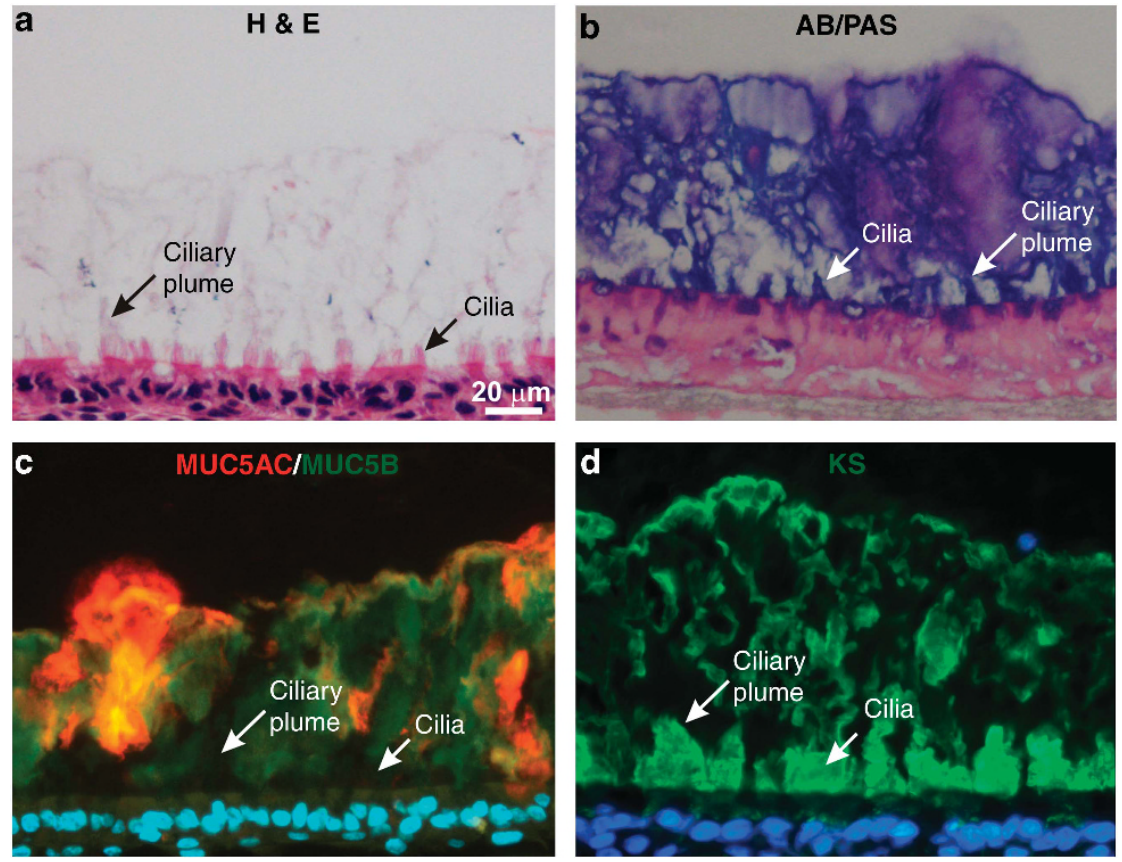

Figure 2 Mucus, mucins, and keratan sulfate (KS) on the mucosal surface of human tracheobronchial epithelial (HTBE) cell cultures. Histological sections of HBTE cell cultures with accumulated mucus secretions were stained for (a) hematoxylin and eosin (H\&E) and (b) alcian blue/periodic acidSchiff (AB/PAS), or immunoprobed with antibodies against (c) MUC5AC (red, monoclonal Ab, 45M1) and MUC5B (green, polyclonal, Cys-rich domains within the mucin repeat domains) or (d) keratan sulfate (green, monoclonal Ab 5D4). Panels $\mathbf{c}$ and $\mathbf{d}$ were counterstained with DAPI. Note height of the accumulated mucus layer $\sim 100 \mu \mathrm{m}$, the intense staining of KS in the periciliary layer, and in all the panels, the plumes of material that appear to extend from ciliary tips and into the mucus (ciliary plumes), while excluding the polymeric mucins. In panel c, intracellular mucins are not apparent, as their fluorescence intensities were too weak to be imaged at gain settings appropriate for the highly intense extracellular signal. For panel $\mathbf{a}, \mathrm{bar}=20 \mu \mathrm{m}$.
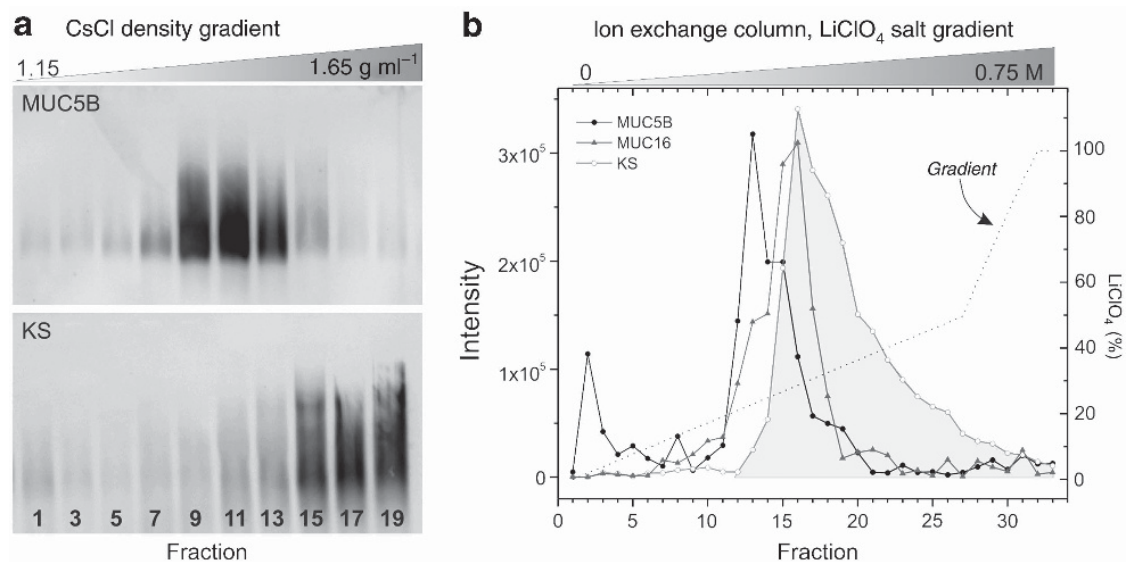

Figure 3 Separation of mucins and keratan sulfate (KS)-conjugated molecules collected in human tracheobronchial epithelial (HTBE) cell culture secretions. Mucus collected from HTBE cell cultures and solubilized in $\mathrm{GuHCl}$ was separated using two different methods. (a) CsCl isopycnic density gradient analysis. Fractions from a $\mathrm{CsCl}$ density gradient following isopynic centrifugation to equilibrium were analyzed by agarose western blotting using antibody probes to MUC5B (top) and KS (bottom). (b) lon exchange column chromatogram. Solubilized mucus was applied to a MonoQ HR 5/5 ion exchange column, after dialysis against urea and disulfide reduction in dithiothreitol. The column was eluted with an increasing gradient of $\mathrm{LiClO}_{4}$ and fractions analyzed by slot blotting, using antibody probes to MUC5B, MUC16, and KS (shown), as well as MUC5AC, MUC1, and MUC4 (not shown). The KS elution profile is highlighted in gray to illustrate that approximately half the KS-positive material elutes with MUC16 (and other mucins), whereas the other half elutes at more acidic $\mathrm{pHs}$.

protein as an approximate index of abundance. The other mucins of interest, MUC5AC, MUC4, and MUC1 were also abundant in the density gradient-separated KS-rich material.
A recently identified mucin, MUC20, which is expressed in the lung, ${ }^{33-35}$ was detected as a single peptide fragment by mass spectrometry of the KS-rich fraction from the $\mathrm{CsCl}$ 
Table 2 Major proteins in KS-rich fractions from HTBE secretions, identified by two dimensional shotgun proteomics

\begin{tabular}{ll}
\hline \multicolumn{2}{c}{ Basis of first separation } \\
\hline $\begin{array}{l}\text { CsCl density gradient } \\
\text { (pooled fractions 17-20) }\end{array}$ & $\begin{array}{l}\text { MonoQ ion-exchange HPLC } \\
\text { (pooled fractions 17-19) }\end{array}$ \\
\hline Mucin-16 & Mucin-16 \\
Mucin-5B & Mucin-5B \\
LPLUNC1 & Dermicidin \\
MUC4 & Mucin-1 \\
Mucin-5AC & WAP domain containing \\
& protein HE4-V2 \\
Complement component C3 & Beta-actin \\
Cytokeratin 9 & LPLUNC1 \\
Beta-actin & DMBT1 \\
WAP domain containing & Cytokeratin 2 \\
protein HE4-V2 & \\
Mucin-1 & \\
Prostate stem cell antigen & \\
PLUNC & \\
\hline
\end{tabular}

Abbreviations: HTBE, human tracheobronchial epithelial; HPLC, high-powered liquid chromatography; KS, keratan sulfate.

The proteins are ranked by the total number of peptides identified for each protein, with higher numbers being consistent with greater abundance.

density gradient. Like MUC5AC and MUC4, it appeared to be absent from the ion exchange separation. A rabbit polyclonal antibody was generated against a synthetic peptide, whose sequence was taken from a portion of the non-glycosylated region of the MUC20 molecule that was detected by mass spectrometry, and was used to probe an agarose western blot of the fractions from the $\mathrm{CsCl}$ density gradient. This analysis showed the antibody to stain a single major band that was concentrated in fractions 15-19 of the gradient (see Supplementary Figure S2 online). Hence, MUC20 has the high buoyant density typical of mucins, one that is distinctly higher than the typical distributions of MUC5B and MUC5AC and within the range in which KS distributes (c.f. Figure 3).

Several globular proteins were also identified in the KS-rich material in both separations (Table 2), but because none of them are known to be highly glycosylated, they are likely complexed with the mucins. The only non-mucin, glycoconjugate identified was DMBT1; a highly $N$ - and $O$-glycosylated protein known to function in mucosal defense as an agglutinin binding to a variety of proteins, including surfactant protein $\mathrm{D}$, lactoferrin, and MUC5B. ${ }^{36}$

\section{Visualizing mucin molecules in mucus from HTBE cell cultures}

Electron and atomic force microscopy were used to visualize and evaluate the diversity of mucin macromolecules in material eluting in the void volume of a Sepharose S-1000 column (Figure 4a,c). Visualization by EM revealed five different dimensional classes of molecules, four of which were "short", linear molecules 190-1,500 $\mathrm{nm}$ long, approximating the predicted sizes of tethered mucins (Table 2). The largest of these molecules $(1,200-1,500 \mathrm{~nm} \times 10-12 \mathrm{~nm})$ had a distinct tail, $\sim 130 \mathrm{~nm}$ in length, with a visual characteristic resembling a "string of beads" (Figure 4a, inset, arrows). This clearly observable structure may represent the SEA-repeat domain region of MUC16, 16 extracellular SEA repeats positioned between the transmembrane domain and the tandem, mucin repeat domains. ${ }^{37-39}$ The extracellular portion of MUC16 is predicted to be at least 22,000 residues in length and if fully extended would make it $\sim 8.8 \mu \mathrm{m}$ long assuming $4 \AA(0.4 \mathrm{~nm}) /$ residue $^{40}$ Hence, a measured contour length of $1-2 \mu \mathrm{m}$ seems reasonable for the molecule detected by EM, as extensive $O$-glycosylation of the extracellular domains combined with a proline-rich content will render the molecule relatively inflexible ${ }^{41}$ and regularly kinked, respectively. The identification of this molecule was confirmed as MUC16 using the well-characterized MUC16 antibody, OC125, which recognizes the SEA domains of MUC16 selectively. ${ }^{38}$ Our visual structural analysis was corroborated using this antibody as immunogold bound exclusively to the end the molecule we predicted represented the SEA-repeat domain of MUC16 (Figure 4 b, arrow).

The second largest class of molecules had similar $10-12 \mathrm{~nm}$ widths as determined for MUC16, but were shorter (750$900 \mathrm{~nm}$ ) and lacked visual evidence of the SEA-repeat domain. Based on the similarity of molecular widths, we tentatively identify these molecules also as MUC16, though whether they represent a fragment of full-length MUC16 or VNTR (variable nucleotide tandem repeat) polymorphic isoforms cannot be determined. The other two classes of linear molecules identified by EM analysis (Figure 4a,c, $\mathbf{c}^{\prime}$ ) were 7-9 $\mathrm{nm}$ wide, and were either 750-900 or 190-200 nm in length (Table 3). We tentatively assign these molecules as MUC4 and MUC1, respectively, based on their predicted lengths relative to MUC16. ${ }^{39}$

The final class of molecule observed (Figure 4c) was identified as the polymeric mucin, MUC5B, based on its visual characteristics ${ }^{18}$ and the dominant abundance of MUC5B in HTBE mucus ( $~ 80 \%$ of the mucins are MUC5B). ${ }^{9}$ Compared with the size of tethered mucins, MUC5B was immense and the structure imaged likely represents a single continuous molecule (indicated by the tracing in Figure $4 \mathbf{c}^{\prime}$ ). The predicted MUC5B molecule exhibited a convoluted contour many micrometers in length and features multiple, crosslinked nodes. Previous studies indicate this molecular form of MUC5B is intermediate in form between a compact, highly crosslinked form resolving at the bottom of the sucrose density gradient, and the linear form resolving at the top. ${ }^{18}$ For comparison, an EM of the fully linearized form of MUC5B is shown in Supplementary Figure S5 online.

Visualization of the glycopeptides representing the gigantic, extracellular glycosylated domains of the MUC16 and MUC4-tethered mucins by atomic force microscopy (AFM) after dispersion on mica revealed the molecules to have similar contour lengths to those measured by EM, but their measured widths are much greater (Figure 4d,e). Molecules identified 

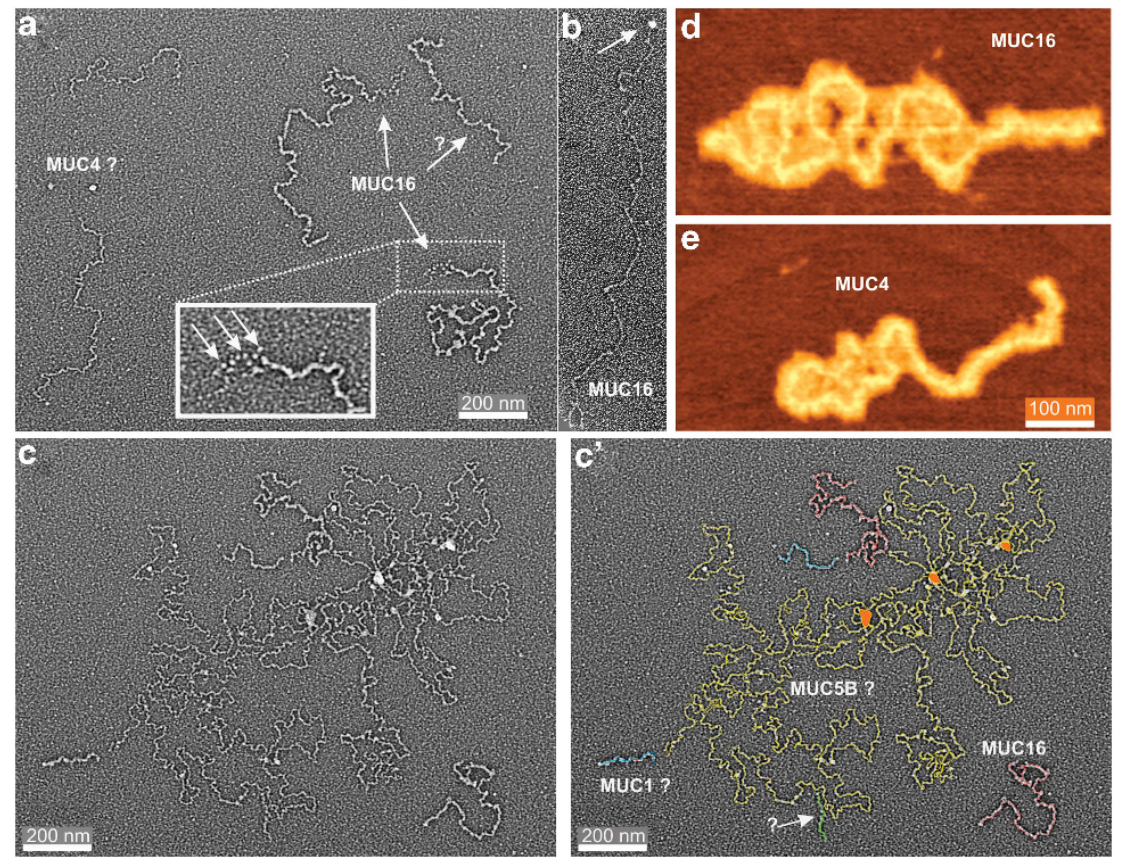

Figure 4 Electron microscopic (EM) and atomic force microscopic (AFM) images of mucins from human tracheobronchial epithelial (HTBE) mucus. Mucins from HTBE cell culture mucus secretions solubilized in phosphate-buffered saline were separated from most of the protein fraction by taking the void volume of a Sephacryl S1000 HPLC (high-powered liquid chromatography) column, and were then fixed in glutaraldehyde and prepared for EM. (a) EM of tethered mucins. MUC16 is identified by the tail at one end of the molecule appearing as a "string of beads" (inset), and MUC4 by relative length and narrower profile. (b) EM of MUC16 labeled with OC125-bound gold bead (arrow). See Experimental Procedures for preparation details. (d and e) AFMs of MUC16 and MUC4. After destructive proteolysis of reduced, polymeric mucins in HTBE mucus, the remaining, large glycosylated extracellular domains of MUC16 and MUC4, which are resistant to proteolysis, were dispersed on mica and visualized by AFM. (c and $\mathbf{c}^{\prime}$ ) EM of tethered and polymeric mucins. Polymeric mucins in samples of HTBE mucins prepared as in panel a were imaged by EM. The mucin profiles in $\mathbf{c}^{\prime}$ were traced with color-coded lines, as indicated. ?=tentative identification.

Table 3 Dimensional classes of linear mucins in mucus from primary HTBE cell cultures observed by electron microscopy (Figure 4)

\begin{tabular}{ll}
\hline Contour length $\times$ width $(\mathbf{n m})$ & Identification \\
\hline $1,200-1,500 \times 10-12$ & MUC16 \\
$750-900 \times 10-12$ & MUC16 $^{a}$ \\
$750-900 \times 7-9$ & MUC4 $^{a}$ \\
$190-200 \times 7-8$ & MUC1 $^{a}$ \\
\hline
\end{tabular}

Abbreviations: HTBE, human tracheobronchial epithelial.

${ }^{a}$ Association tentative.

as MUC16 or MUC4 had widths by AFM of 50-60 and 30$35 \mathrm{~nm}$, respectively, or approximately fivefold wider than their appearance by EM (c.f. Table 3). This difference is presumably due to better maintenance of the hydration state of the mucins visualized by AFM-molecules visualized by EM are subjected to strong dehydration forces imposed by the alcohol washings and the vacuum required for rotary shadowing.

\section{Localization of tethered mucins in airway epithelia}

Based on the identification of KS-associated high MW glycoconjugates on the surface of HTBE cultures as tethered mucins, we probed histological sections of HTBE and human bronchial epithelia with antibodies to specific mucins to determine their subcellular localization. Probing sections for an extracellular epitope of MUC1 (B27.29 $\mathrm{mAb}^{42}$ ) showed that the mucin localized extensively within the PCL at the base of the cilia and to basal epithelial cells (Figure 5a, left and right panels). At this resolution, MUC1 appeared absent from upper regions of the cilium, if not from the entire ciliary shaft.

By contrast, probing sections with a polyclonal antibody raised to an extracellular peptide of MUC4 (MUCH4), whose sequence was identified initially by mass spectrometry, localized MUC4 immunoreactivity to the ciliary shafts (Figure $\mathbf{5 b}$ ). In HTBE cultures with accumulated mucus secretions, MUC4 was also detected in the ciliary plumes (Figure $\mathbf{5 b}$, left panel). An alternative MUC4 monoclonal antibody, Ig8, revealed robust expression of MUC4 in ciliated cells (see Supplementary Figure S1B online). This antibody failed to detect extracellular MUC4 as the antibody recognizes a peptide sequence in the VNTR mucin domain of MUC4, a heavily glycosylated region of MUC4 likely to sterically hinder antibody access to the peptide epitope. Further analysis immunodetecting MUC1 and MUC4 together in the same preparation showed spatial co-localization in the cell surface regions corresponding to the microvilli of ciliated cells at the base of the cilia 

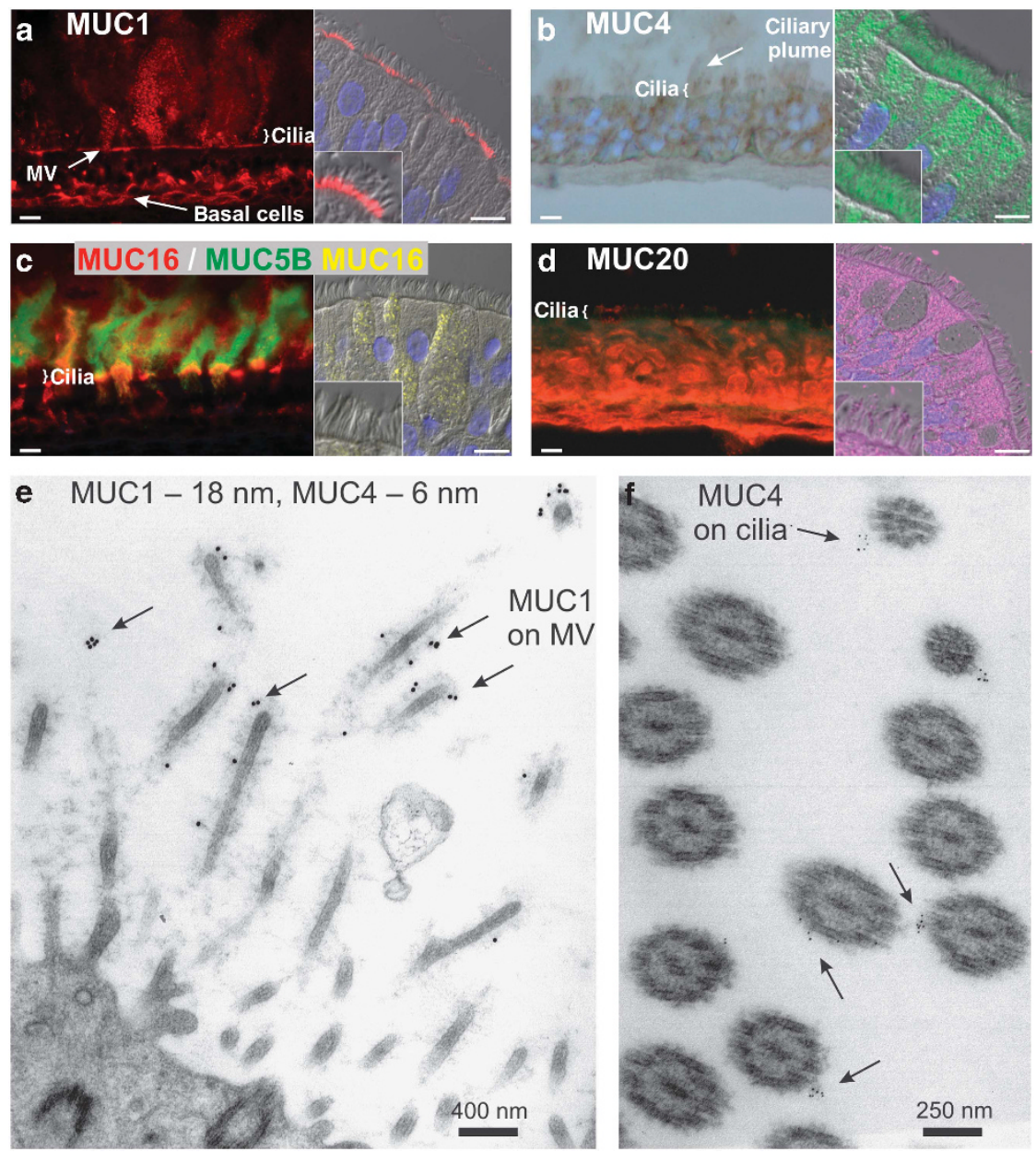

Figure 5 Localization of tethered mucins in airway epithelia. Panels a-d show staining by immunofluorescence (IF) for MUC1, MUC4, MUC16, and MUC20, as indicated. The left and right hand image of each pair within a panel shows immunolocalization in a human tracheobronchial epithelial (HTBE) cell culture and in human bronchial epithelium, respectively. The HTBE cultures in panels a-c had accumulated mucus, per Figure 2. For panel b, note that the antibodies used in the two images were to the same peptide sequence (MUCH4), but represented different polyclonal antibodies, one of which was suitable for immunohistochemistry, the other for IF. Bars $=10 \mu \mathrm{m}$. Panels $\mathbf{e}$ and $\mathbf{f}$ show MUC1 and MUC4 localization of human bronchial epithelium by immuno-electron microscopy (EM). The tissue was exposed simultaneously to MUC1 and MUC4 antibodies conjugated to 18 and $6 \mathrm{~nm}$ gold beads, respectively, then washed extensively, fixed, and prepared for conventional EM. Arrows indicate gold beads labeling the glycocalyx of microvilli (MV) and cilia.

(see Supplementary Figure S2C online). However, within the limits of resolution of light microscopy, it is possible that this apparent overlap might arise from an intercalation of mucin molecules within the glycocalyx, but with different points of attachment, e.g., microvilli and cilia.

To clarify the spatial localization of MUC1 and MUC4, immuno-EM was used to identify the ultrastructural localization of MUC1 and MUC4 immunoreactivity. Figure 5e,f show immunolocalization of MUC1 (214D4 mAb conjugated to $18 \mathrm{~nm}$ gold beads) in glycocalyx structures associated with microvilli, while MUC4 (MUCH4 antibody conjugated to $6 \mathrm{~nm}$ gold beads) immunolocalized to glycocalyx associated with cilia. In 11 different electron micrographs, we enumerated 366 MUC1conjugated and 129 MUC4-conjugated beads, of which 93.7 and $90.7 \%$ were associated with the microvilli or cilia, respectively. Approximately $\sim 6 \%$ of beads were attached to cell surface elements not identified. Together, these immunolocalization data indicate a differential distribution of MUC1- and MUC4tethered mucins on ciliated cells, with MUC1 preferentially present on microvilli and MUC4 preferentially on ciliary shafts.

The cellular distribution of MUC1 and MUC4 in airway epithelium also differed. Although MUC1 and MUC4 localized to basal epithelial cells, MUC4 was more robustly expressed than MUC1 in ciliated cells (Figure 5a,b, Supplementary Figures S1B,C, S3A,B online). However, in HTBE cell cultures with accumulated mucus, MUC1 and MUC4 were both detected within the mucus secretions (Figure 5a,b). Although MUC1 has been reported to be secreted/shed from airway epithelium, ${ }^{39}$ we recently described the presence of MUC1 and MUC4 on exosome-like vesicles shed from the airway surface into the mucus secretions, thus contributing to the presence of these tethered mucins in accumulated mucus secretions. ${ }^{43}$ Discerning whether MUC1 and MUC4 immunoreactivity 
in mucus secretions represents shed MUC1/4-containing vesicles, shed mucin molecules per se or both is beyond the resolution of our current fluorescent microscopy studies.

MUC16, identified by both OC125 and X325 antibodies, was predominately expressed by goblet cells in the surface epithelium (Figure 5c and Supplementary Figures S3 and S4 online). The OC125 antibody robustly detected the apical membrane of goblet cells, but stained cytoplasm of these cells only lightly. The X325 antibody, which recognizes an extracellular epitope distinct from the OC125 epitope, ${ }^{44}$ stained cytoplasmic secretory granules to a greater degree than OC125. Interestingly, in HTBE cell cultures with accumulated mucus secretions, MUC16 capped goblet cells, which co-localized with MUC5B immunodetection. Indeed, in addition to capping MUC5Bcontaining/secreting cells, MUC16 also emanated from the goblet cells in broad pillars of immunoreactivity, suggesting co-release of MUC16 with MUC5B (Figure 5c, left). MUC16 was not immunodetected at the level of the cilia or ciliated cells and may be excluded from these domains as suggested for MUC5AC and MUC5B exclusion.

Using the antibody generated against MUC20 as described above (see Supplementary Figure S2 online), we localized MUC20 immunoreactivity to the cytoplasm of ciliated and basal epithelial cells and to the cilia (Figure 5d and Supplementary Figure S3D online). As MUC20 was identified by proteomics in mucus secretions of HTBE cultures, it is likely MUC20 is secreted/shed from the epithelium into airway secretions.

Tethered mucins were also detected in the submucosal glands of human tracheobronchial airway tissue. MUC1 was immunolocalized to a sub-population of acinar cells (see Supplementary Figure S1D online), and MUC16 immunoreactivity was associated with the surface of mucous acinar cells containing MUC5B (see Supplementary Figure S1E online), similar to the association observed in the surface epithelium of HTBE cell culture (Figure 5c). MUC20 immunoreactivity was localized in all acinar cells (see Supplementary Figure S1F online). MUC4 was not detectable in submucosal glands.

\section{Visualization of the glycocalyx}

The classical view of the mucus and PCLs on airway surfaces is that both layers are distinct and thixotropic-type forces generated by the beating cilia prevents penetration of secreted mucins into the PCL. ${ }^{13}$ This perception of a "watery" PCL to enable effective ciliary beating was formed by the lack of visible materials within the periciliary spaces. Conventional histochemical and EM techniques to visualize glycocalyx structure and components such as the metallic stains colloidal iron and ruthenium red likely collapse the molecular structure and network of the glycocalyx via electrostatic interactions. ${ }^{20,22,45}$ These artifacts of tissue preservation and visualization have led to the perception that the airway glycocalyx is a layer at the cell surface, distinct from the PCL (e.g., ref. 46). Our previous ${ }^{20}$ and current studies, however, indicate a rich population of tethered mucins populating periciliary surfaces that could well serve as components of a selective filter, which excludes large molecules, molecular complexes, and microbes.

To test this notion, we examined the ultrastructure of the airway surface using conventional and rapid freeze/freeze substitution (RF/FS) techniques. Figure 6a shows the ultrastructure of the mucosal surface of human bronchial epithelium after staining with ruthenium red and conventional processing for EM. Notably, the glycocalyx is seen as a thin, fuzzy layer on the surfaces of the microvilli and is hardly visible on cilia shafts. Although strands of material appear to project between microvilli, the overall periciliary microenvironment is seen as open space using this technique. In mouse tracheal airways ex vivo, ruthenium red staining revealed a much more robust and dense glycocalyx (see Supplementary Figure S6 online), but even in this tissue, "open space" predominates over evidence of a complex molecular matrix. Using histological techniques known to better preserve native carbohydrate structure, i.e., RS/FS, glycocalyx structure is revealed as a complex, finely textured matrix filling the available space within the PCL (Figure 6b,c). In Figure 6b, the glycocalyx matrix visually fills spaces between cilia shafts, and Figure $\mathbf{6 c}$ shows the glycocalyx of a goblet cell, with cilia projected above filled with a fine grained, fibrillar matrix material-notably the matrix occupies spaces $>1 \mu \mathrm{m}$. A magnified view of Figure $\mathbf{6 c}$ (lower panel), shows matrix fibrils radiating from microvilli cut in cross-section, and in both panels where the structures are cut longitudinally, the fibrils form a lattice with an irregular, honeycombed structure.

Our previous and current studies identify an underappreciated, rich population of tethered, highly glycosylated mucins within the PCL populating the epithelial cell surface glycocalyx. These structurally defined and diverse molecules are likely important in innate protection of the airways surfaces. They likely influence appropriate airway surface hydration for effective mucus clearance as well as functioning as an innate immunological molecular sieve limiting the access of molecules and molecular complexes, including pathogenic particulates and microbes ${ }^{20}$ to the epithelial cells and tissues underlying the glycocalyx. In an initial attempt to test this notion, we used several human viruses as molecular probes of particles with defined size and structure to determine the penetration of these organisms through the glycocalyx layer. Notably, the sizes of the viral particles used, 30 and $100 \mathrm{~nm}$, are less than the typical, $\sim 250 \mathrm{~nm}$ radius of gyration for polymeric mucins. Figure 7 shows reasonable penetration of adenoasociated virus $(30 \mathrm{~nm})$ into the glycocalyx of ciliated cell microvilli, whereas the larger adenovirus particle $(100 \mathrm{~nm})$ were excluded. It is likely the glycocalyx and the heavily glycosylated glycoconjugates contained within act to restrict particle access based on size, as well as biochemical structure and interactions.

\section{DISCUSSION}

We provide evidence of the spatial molecular structure and distribution of soluble mucins (mucus) and tethered mucins (PCL) on the mucosal surface of human airway epithelium 

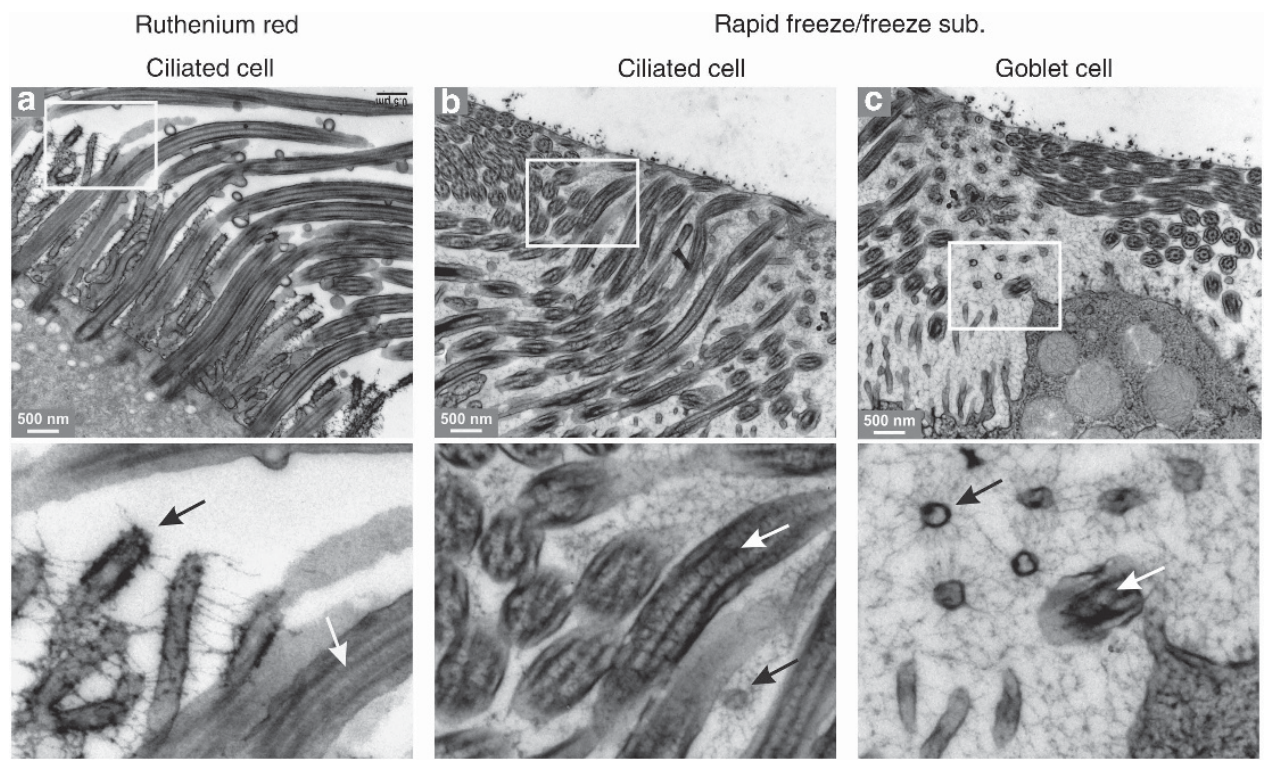

Figure 6 Comparison of fixation methods on mucosal glycocalyx in human bronchial epithelium. Bottom panels are magnified portions of the images above, as indicated by the white boxes. (a) Tissue stained with ruthenium red and processed for conventional electron microscopy. Note how ruthenium red causes the glycocalyx to appear as thick, sparse strands, leaving most of the intercellular space apparently open. (b and c) Tissue fixed by rapid freezing and freeze substitution (RF/FS), showing areas of the periciliary layer above ciliated and goblet cells, as indicated. Note the finely textured, space filling matrix revealed by RF/FS. Lower panels, black arrows = microvilli, white arrows=cilia. See text for more details.

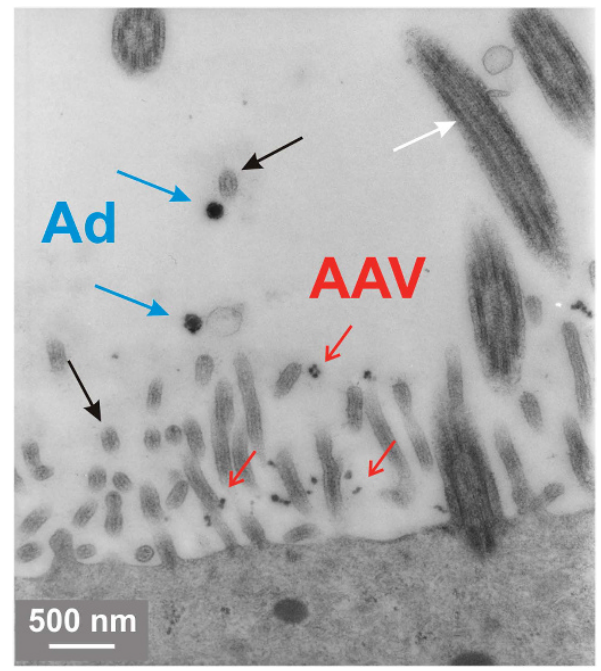

Figure 7 Interaction of viral particles with the glycocalyx of the periciliary layer. Human tracheobronchial epithelial cell cultures were incubated mucosally with particles of unlabeled adenovirus (Ad, $100 \mathrm{~nm}$, blue arrows) and adenoassociated virus (AAV, $30 \mathrm{~nm}$, red arrows), then rinsed, fixed, and prepared for electron microscopy. White arrow indicates a cilium; black arrow, a microvillus.

that is consistent with early descriptions of the two separate gel domains of the mucociliary clearance system. ${ }^{4}$ In our studies of well-characterized HTBE cultures, we show that MUC5AC and MUC5B were clearly excluded from the PCL (Figure 2c), despite polymeric mucins being of sufficiently small molecular size to enter the $0.1-1 \mu \mathrm{m}$-sized spaces around and between the cilia (Figure 1). These inter-ciliary spaces, however, are not devoid of glycosylated material as KS immunoreactivity in the PCL was robust (Figure 2). KS is a glycosaminoglycan commonly associated with glycoconjugates; hence, KS localized in the PCL associated with cilia and ciliary plumes suggested that KS is a major component of a significant glycocalyx structure within the PCL. Historically, the microvilli on the apical surfaces of ciliated cells have been shown to be coated in acidic mucosubstances, most likely highly sulfated glycoconjugates. ${ }^{30}$ In connective tissues such as cartilage, KS is associated with proteoglycans, e.g., aggrecan, but in recent years KS has also been found conjugated to mucins, ${ }^{26,27}$ including those on the mucosal surfaces of human airways. ${ }^{28}$ The airway mucins conjugated to KS have not been identified. Examination of the separation profiles of airway mucus analyzed by both $\mathrm{CsCl}$ isopycnic density centrifugation and ion exchange HPLC (high-powered liquid chromatography) showed that KS immunoreactivity overlapped significantly with the airway soluble mucins, MUCs 5AC,and MUC5B, and the tethered mucins, MUC1, MUC4, and MUC16 (Figure 3). These biochemical associations were confirmed by mass spectroscopy. The most abundant proteins identified in KS-rich fractions ranked by peptide coverage were MUC16, MUC5B, MUC1, and MUC4 (Table 2). MUC20 was less abundant but was also identified in the KS-rich material. No proteoglycans were identified, consistent with our earlier findings. ${ }^{7}$ All attempts to immunoprecipitate KS immunoreactive materials were hindered by non-specific binding of the KS-rich materials to immunoprecipitation beads. This finding was reminiscent of the known interactions of mucins with beads, with mucin molecules forming strands and bundles around beads. ${ }^{47,48}$ Together these data indicate that both the polymeric and tethered mucins identified 
in the KS-rich fractions of airway mucus were conjugated to KS and MUCs 1, 4, 16, and 20 are largely responsible for the robust KS immunoreactivity within the PCL (Figure 2 and Supplementary Figure S1 online).

Immunolocalization studies focused on reagents directed to the extracellular, non-glycosylated domains of the tethered mucins revealed a strong spatial organization of these molecules within the PCL (Figure 5 and Supplementary Figures S1, S3, and $\mathbf{S} 4$ online). MUC1 immunoreactivity was localized predominately to microvilli of ciliated cells, while MUC4, and to a lesser extent MUC20, localized predominately to the cilia shafts. By contrast and as reported originally, ${ }^{49} \mathrm{MUC} 16$ was predominately localized to the apical membrane and secretory granules of goblet cells (and mucous cells of submucosal glands). The tethered mucins were also present in the mucus layer, presumably after being shed from the cell surface (Figure 5a-c). In the mucus layer, MUC1 immunoreactivity was associated with vesicular structures, likely the exosome-like vesicles we have previously shown to be rich in MUC1. ${ }^{43}$ MUC4 and MUC16 immunoreactivity were also present in the mucus layer, associating with MUC5AC and MUC5B.

The ciliary plumes observed in mucus secretions in HTBE cell cultures (Figures $\mathbf{2}$ and 5 ) have not been previously described. Plumes stain with alcian blue/periodic acid-Schiff and hematoxylin and eosin, and are immunoreactive to KS and MUC4 antibodies. They have a filamentous appearance, excluding MUC5AC and MUC5B mucins as they project into the overlying mucus layer from attachment points on ciliary tips (Figures 2 and 5). In a recent study, we observed transient, physical interactions between ciliary tips and polymeric mucins, interactions that appeared to be important to mucus transport. ${ }^{48}$ We speculate that the ciliary plumes seen in accumulated mucus, in vitro, are important to the cilia-mucin interactions in mucus transport under more physiologically relevant conditions, in vivo.

These studies highlight the importance of ultrastructural preservation of glycocalyx for assessing components of the PCL. Although the glycocalyx layer has been appreciated for many years, the abundance and barrier function of this layer in airways has largely been underappreciated in conventional EM studies that rely on the use of metal-based dyes such as colloidal iron and ruthenium red. Visualization of the glycocalyx by these techniques rely on rendering the glycocalyx electron dense, but these reagents also collapse the molecular architecture of the glycocalyx structure electrostatically (see ref. 50). As demonstrated dramatically for the glycocalyx of vascular endothelium, when the specimen is prepared instead by RF/FS, the glycocalyx is revealed to be much more complex than when visualized by conventional EM, and in this particular case, it is around $10-11 \mu \mathrm{m}$ in thickness. ${ }^{22}$ Previously, RF/FS EM techniques have revealed that the glycocalyx of the airway epithelium in HTBE and ex vivo human tracheal epithelium is a complex mesh-like glycocalyx restricting the access of adenoviral particles to the apical membrane of columnar airway epithelium; ${ }^{20}$ our new data suggest that the glycocalyx may also prevent the penetration of mucus into the PCL.
Figure $\mathbf{6 b}, \mathbf{c}$ show RF/FS EMs of HTBE when combined with high-contrast digital photography reveals an even denser glycocalyx than earlier described: the meshwork of the glycocalyx, with interstices of $<100 \mathrm{~nm}$, clearly fills the interciliary spaces in the PCL over distances measuring in the $\mu \mathrm{m}$ range. Even by these EM techniques, one assumes that this mesh-like ultrastructure would tend to exclude the polymeric mucins, which have radii of gyration $>200 \mathrm{~nm} .{ }^{17,18}$ Consistent with this possibility, a recent study by colleagues, Button et al. ${ }^{51}$, found that within the PCL labeled dextrans and other probes of defined sizes were excluded progressively from the cell surface of ciliated cells in HTBE cell cultures: molecules with radii of gyration $>40 \mathrm{~nm}$ were excluded from the PCL, and those $>18 \mathrm{~nm}$ were unable to penetrate through the microvilli to the cell surface, whereas probes $<5 \mathrm{~nm}$ penetrated all the way to the cell surface.

In addition to biophysical exclusion from the PCL, another factor contributing to the separation of PCL and mucus layers may be the nature of the mucus gel, per se. The polymeric mucins are known to interact with $>100$ other proteins, many of which have known functions in innate defense, and $~ 30 \%$ of them bind directly to the mucins (refs. 7,10; and unpublished observations). The close association of these proteins to the polymeric mucins would likely limit their diffusion and while their specific functions remain to be elucidated, we note that they are potentially responsible, in part, for the transient crosslinks between mucin molecules that impart elasticity to the gel. ${ }^{52}$ These interactions would likely also restrain mobility of mucin molecules, potentially constraining them to the mucus layer.

An important question for the role of tethered mucins in imparting the mesh-like properties of the glycocalyx in the PCL is related to whether tethered mucins identified in this study are large enough to occupy the spatial environment of the interciliary spaces. The tethered mucins, MUC1, MUC4, and MUC16, are molecularly massive in relation to globular proteins, with molecular masses ranging from several hundred $\mathrm{kDa}$ to $2.5 \mathrm{MDa}$. Most of the mass of mucins is attributed to the high density of $\mathrm{N}$ - and $\mathrm{O}$-linked glycans attached to the peptide backbone. Tethered mucins are predicted to exhibit linear and rigid structures resembling "bottle-brushes", highlighting the extent to which these molecules may project into extracellular spaces. The extracellular domains of MUCs 1,4 , and 16 range from approximately 1,500 to 22,000 residues in length with genetic variation in the number of tandem repeats dictating the absolute length (see refs. 39,53). In contrast to MUCs 1, 4, and 16 , the external domain of MUC20 is substantially shorter, with only 425 residues extending into the extracellular space. ${ }^{33}$ Using the persistence length of $4 \AA(0.4 \mathrm{~nm}) /$ residue for a maximally extended peptide, ${ }^{40}$ the maximum possible lengths calculated for the tethered mucins are MUC20-170 nm, MUC1-600 nm, MUC4-3,380 nm, and MUC16-8,800 nm. Although the actual lengths will likely be shorter, considering the nonglycosylated and glycosylated domains will possess globular and linear, rigid structures, respectively, the contour lengths of tethered mucins isolated from HTBE cell mucus ranged from 
190 to $1,500 \mathrm{~nm}$, within which there were four recognizable size categories assignable to MUCs 1, 4, and 16 (Figure 4, Table 3). Using EM analysis, the tethered mucins were 7-12 nm in breadth, but MUC4 and MUC16 when visualized in the more hydrated state allowed by AFM measured approximately 30 and $55 \mathrm{~nm}$ wide (Figure 4). Hence, the tethered mucins represent very large molecules fully capable of forming the dense meshwork of the glycocalyx in the interciliary spaces of the PCL (Figure 6).

Although our studies have revealed MUCs 1, 4, 16, and 20 to be the predominant tethered mucins on the airway surface, other tethered mucins have also been identified at the mRNA level in human and mouse airway epithelia, e.g., MUC13, MUC15, and, MUC3..$^{20,34,54,55}$ Currently, reagents to detect the mucins encoded by these genes in human tissues are not available but these mucins could significantly contribute to the glycocalyx mesh within the PCL. Other glyconjugates, such as hyaluronan, ${ }^{28}$ as well as unidentified glycoproteins or glycoconjugates are likely to add to the complexity of the glycocalyx mesh structure.

The intriguing findings that the PCL does not represent a "watery" aqueous layer but rather a highly dense gel-like meshwork, it may be expected that the tethered mucins in the PCL impart a viscous drag which interferes with ciliary beating and effective mucus transport. However, studies investigating the physics of polymers attached to surfaces have shown that grafted polyelectrolyte brushes attached to surfaces offer remarkably low frictional interactions between opposing interfaces. ${ }^{56,57}$ Hence, the tethered mucins of the glycocalyx, as grafted polyelectrolyte brushes, may not only pose a barrier to the penetration of the PCL, but may also confer a lubricating property to facilitate ciliary beating to ensure effective mucus transport.

In conclusion, the polymeric and tethered mucins on the airway surface are organized into two distinct layers, with the polymeric "transporting" mucins apparently excluded from the PCL by the tethered mucins forming the glycocalyx that decorates the cilia, microvilli, and apical surfaces of the epithelial cells. Figure 8 summarizes these findings, showing the mucociliary clearance system diagrammatically with a separation of the layers and the locations/sources of the mucin molecules. The polymeric mucins, MUC5AC and MUC5B, predominate in the mucus layer, while membrane-bound mucins, MUCs 1, 4, 16, and 20 also contribute to the mucus layer after shedding from the cell surfaces. Although MUC1 is associated primarily with exosome-like vesicles within the mucus layer, MUC4 localizes primarily to ciliary plumes, and MUCs 4,16 , and 20 are dispersed within the mucus layer. The PCL is composed mainly of large membrane mucins, MUC4 and MUC16, with MUC4 tethered predominately to cilia and MUC16 to the apical membrane of goblet cells. MUC1, tethered to microvilli, serves to strengthen the protective matrix at the base of cilia. We speculate that the glycocalyx matrix in its entirety dominates the physical properties of the PCL. The matrix (i) provides dynamic support for the over-lying mucus gel, (ii) acts as a barrier to the penetration of particulates, pathogens, and polymeric mucins, and (iii) facilitates ciliary beating by lubricating the interface between neighboring cilia and possibly acting as molecular springs,

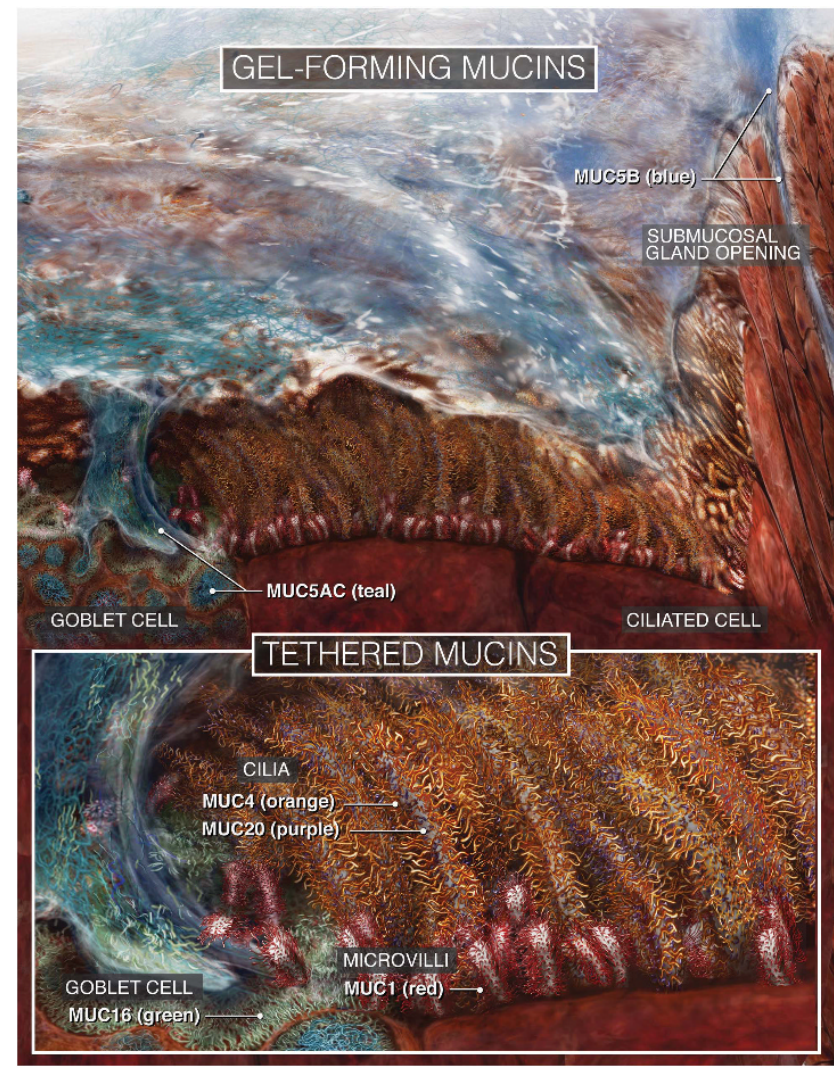

Figure 8 Schematic depicting a scaled view of the mucosal surface of human airways. Top: overview of the mucociliary apparatus, showing a ciliated airway mucosal surface covered with a sheet of mucus. For clarity, the mucus sheet is depicted as a thin layer; in vivo, it would be substantially thicker. Bottom: higher magnification views of a goblet cell (left), cilia, and the periciliary layer, showing the molecular organization of the different mucins.

which, alternately compressing and relaxing, transiently store and release energy during the ciliary beat cycle. The multilayer protection model proposed at the airway lumen modifies our view of the structure, organization, and role of these layers as well as increasing the significance of the glycocalyx mesh in maintaining mucus homeostasis, mucus transport, and airway innate defense. As such these studies represent a paradigm shift by which the mucins participate directly in innate lung defense through a highly ordered molecular structure. Strategies to strengthen this barrier against potential pathogens or enhance penetration of potential therapeutics may identify new technologies to treat airway diseases.

\section{METHODS}

Human tracheobronchial epithelium was obtained from airways resected from normal donor tissue from the University of North Carolina (UNC) lung transplant program or from NDRI (National disease research interchange) under UNC Institutional Review Board-approved protocols. Mouse tracheal epithelium was obtained by tissue harvest, under protocols approved by the UNC Institutional Animal Use and Care Committee.

Cell culture and collection of mucus. HTBE cells were isolated by the UNC Cystic Fibrosis Center Tissue Culture Core and expanded 
on plastic to generate passage 1 primary cells, which were plated at a density of $600 \mathrm{k}$ cells per well on permeable Transwell-Col (T-Col, $24 \mathrm{~mm}$ diameter) supports. ${ }^{58}$ Once polarized, cells were cultured at an air-liquid interface for 4-6 weeks to form well-differentiated cultures resembling in vivo pseudostratified mucociliary epithelium. ${ }^{58,59}$ Mucus secretions were obtained from mature HTBE cell cultures by incubating $1 \mathrm{ml}$ of phosphate-buffered saline on the apical surface of the cultures for $30 \mathrm{~min}$ at $37^{\circ} \mathrm{C}$ and then harvesting the phosphate-buffered saline with a large caliber pipette. Harvest was repeated twice to obtain $2 \mathrm{ml}$ of phosphate-buffered saline-diluted mucus per culture and then solubilized with solid $\mathrm{GuHCl}$ to make a $4 \mathrm{M}$ solution. The mucus samples were centrifuged at $300 \times g$ for 10 min to remove debris and pooled with other samples collected under identical conditions.

Histology: immunohistochemistry and immunofluorescence. HTBE cultures were prepared for examination by light microscopy using two methods. The first method was designed to preserve accumulated mucus on the culture mucosal surfaces. Cultures were maintained for 5-7 days without apical surface washing to allow mucus to accumulate. These cultures were then fixed in an alcohol-based fixative (Omnifix, FR Chemical, Mt. Vernon, NY) to preserve the thick mucus layer. ${ }^{25}$ The second method fixed cultures in $4 \%$ paraformaldehyde to enable conventional histochemistry. In both methods, paraffin-embedded histological sections were prepared and immunostained as previously described ${ }^{27}$ using monoclonal mouse IgG antibodies specific against MUC1 (B27.29; 115D8, kind gifts from Fujirebio Diagnostics, Malvern, PA), MUC5B (EU5B, ${ }^{8}$ MUC16 (OC125, X325), and keratan sulfate (5D4, Seikagaku, Tokyo, Japan), followed by goat anti-mouse AlexaFluor 594 (Invitrogen, Grand Island, NY), or polyclonal antibodies against MUC4 (MUCH4, peptide sequence: FLNSNSGLQGLQFYR), MUC5AC (LUM5-1), ${ }^{8}$ and MUC20 (MUCH20, peptide sequence: LSVASPEDLTDPR), followed by goat anti-rabbit AlexaFluor 594 (Invitrogen). Images were taken with Leica SP2 Laser Scanning Confocal, or DM IRB conventional widefield microscopes, and processed with Adobe Photoshop CS2. The antibodies used are summarized in Table 1.

RF/FS and ruthenium red protocols. RF/FS was performed as described previously. ${ }^{20}$ Briefly, samples of human airway epithelium dissected from the same patient source used for the generation of HTBE cell cultures were immersed in ice-cold $0.2 \mathrm{M}$ sucrose solution $(100 \mathrm{ml}$ of $0.2 \mathrm{M}$ Sorenson's buffer, $100 \mathrm{ml}$ of distilled water, and $13.6 \mathrm{~g}$ of sucrose) for $1 \mathrm{~h}$ at $4{ }^{\circ} \mathrm{C}$, then in ice-cold $25 \%$ glycerol solution $(3.8 \mathrm{ml}$ of $0.2 \mathrm{M}$ Sorenson's buffer, $3.8 \mathrm{ml}$ of distilled water, and $2.5 \mathrm{ml}$ of glycerol) for no longer than $1 \mathrm{~h}$, and then plunge-frozen in Freon, and stored in liquid $\mathrm{N}_{2}$. The samples were transferred to cooled $4 \%$ osmium tetroxide in acetone, stored at $-80^{\circ} \mathrm{C}$ for 4 days, and then transferred to a $-20^{\circ} \mathrm{C}$ freezer for $2 \mathrm{~h}$, then a $4^{\circ} \mathrm{C}$ refrigerator for $2 \mathrm{~h}$, then held at room temperature for $1 \mathrm{~h}$. After an acetone wash, the samples were infiltrated with propylene oxide and embedded in Epon resin for standard transmission electron microscopic processing.

For the visualization of the glycocalyx on the cilia by conventional EM, samples were immersed in a glutaraldehyde solution ( $5 \mathrm{ml}$ of $4 \%$ glutaraldehyde, $5 \mathrm{ml}$ of $0.2 \mathrm{M}$ cacodylate buffer, and 1,500 p.p.m. of ruthenium red) for $1 \mathrm{~h}$ at room temperature. After rinsing in $0.2 \mathrm{M}$ cacodylate buffer, the tissues were immersed in an osmium tetroxide solution $(5 \mathrm{ml}$ of $5 \%$ osmium tetroxide, $5 \mathrm{ml}$ of $0.2 \mathrm{M}$ cacodylate buffer, and $5 \mathrm{ml}$ with 1,500 p.p.m. of ruthenium red) for $3 \mathrm{~h}$ at room temperature. After a rinse in $0.2 \mathrm{M}$ cacodylate buffer, standard processing for transmission EM was performed.

$\mathrm{CsCl} / 4 \mathrm{M} \mathrm{GuHCl}$ density-gradient centrifugation. $\mathrm{CsCl}$ was added to collected mucus in $4 \mathrm{M} \mathrm{GuHCl}$ to a density of $1.45 \mathrm{~g} \mathrm{ml}^{-1}$, and isopycnic density-gradient centrifugation was performed for $60 \mathrm{~h}$ at 36,000 r.p.m. on Beckman L8-M ultracentrifuge using $50.2 \mathrm{TI}, 12 \times 40 \mathrm{ml}$ rotor. Samples were unloaded as $2 \mathrm{ml}$ fractions from the top and aliquots analyzed for absorbance at $280 \mathrm{~nm}$ (total protein), density, and, after transfer to a nitrocellulose membrane by slot blotting, reactivity with periodic acidSchiff and antibodies to MUC5B and KS.

Characterization of mucin species by HPLC ion-exchange chromatography. Mucus samples in $4 \mathrm{M} \mathrm{GuHCl}$ were dialyzed into urea reduction buffer (6M urea containing $0.1 \mathrm{M}$ Tris $/ 5 \mathrm{mM}$ EDTA, $\mathrm{pH}$ 8.0) and treated with $10 \mathrm{mM}$ dithiothreitol for $2 \mathrm{~h}$ at $37^{\circ} \mathrm{C}$. Iodoacetamide was added to a final concentration of $25 \mathrm{mM}$, and the mixture was left in the dark for $30 \mathrm{~min}$ at room temperature. The reduced samples were chromatographed on an Amersham Biosciences (Piscataway, NJ) MonoQ HR 5/5 column, using Amersham Biosciences, Ettan LC high-pressure chromatography system. The column was eluted with a linear gradient of $0-0.75 \mathrm{M}$ lithium perchlorate $/ 10 \mathrm{mM}$ piperazine in $6 \mathrm{M}$ urea $(\mathrm{pH}$ 5.0 ) with a flow rate $1 \mathrm{ml} \mathrm{min}^{-1}$, and $0.5 \mathrm{ml}$ fractions were collected. Aliquots from the fractions were transferred to a nitrocellulose membrane by slot blotting and tested for reactivity with monoclonal antibodies against MUC1, MUC16, and KS, and with polyclonal antibodies against MUC5AC, MUC5B, and MUC4 using a standard chemiluminescence assay. The relative intensity of the slot blot bands was measured by densitometry and quantitated by ImageQuant (Molecular Dynamics, Sunnyvale, CA) software.

Agarose gel electrophoresis/western blotting. To determine the distribution and charge dispersion of mucins over the $\mathrm{CsCl}$ density gradient, agarose gel electrophoresis was performed under reducing conditions in $1 \%(\mathrm{w} / \mathrm{v})$ agarose as described previously. ${ }^{60}$ Mucins were separated and western blotted onto nitrocellulose membranes by vacuum transfer, then probed for mucins with antibodies specific for MUC5B, MUC5AC, KS, MUC1, MUC4, and MUC16 (Table 1). Immunodetection was performed using infrared dye-labeled secondary antibodies and visualized using a Li-Cor Odyssey infrared detection system according to the manufacturer's protocol (Li-Cor Biosciences, Lincoln, NE).

Mass spectrometry and multi-angle laser-light scattering. Mass spectrometry for the analysis of mucus fractions was performed essentially as described previously. ${ }^{7}$ Mucin-containing pools from the density gradient centrifugation were reduced, alkylated, and trypsin digested. The resulting peptides were subjected to nano-LC-MS/MS (liquid chromatography-tandem mass spectrometry) analysis. All data were acquired using Waters Q-Tof micro, hybrid quadrapole orthogonal acceleration timeof-flight mass spectrometer (Waters, Manchester, UK) with the manufacturer's MassLynx 4.0 software. The data processed were searched against updated NCBInr and Swiss-Prot databases using the Mascot search engine.

Multi-angle laser-light scattering was performed in-line with gel permeation chromatography to measure the weight average MW and hydrodynamic radius of gyration distributions of isolated whole, and tryptically digested MUC4 and MUC16 mucins. This approach yields absolute values of concentration, and MW and size distributions. ${ }^{52}$ Briefly, the mucin preparations were chromatographed on a Sephacryl 1000 or Sepharose CL-2B (GE Healthcare Biosciences, Pittsburgh, PA; $15 \mathrm{~cm} \times 2.5 \mathrm{~cm}$ ) column eluted with $0.2 \mathrm{M} \mathrm{NaCl}$ at a flow rate of $500 \mu \mathrm{min}^{-1}$. The column effluent was passed through an in-line Dawn DSP laser photometer coupled to an Optilab 903 interferometric refractometer (both from Wyatt Technology, Santa Barbara, CA) to measure light scattering and sample concentration, respectively. Light scattering measurements were taken continuously at 18 angles between $15^{\circ}$ and $151^{\circ}$; the data were analyzed using Wyatt Technology's ASTRA software.

EM of mucins. Mucins obtained from HTBE mucus collected in phosphate-buffered saline were isolated as the void volume eluting from a Sephacryl 1000 (S1000) HPLC column. The mucins were adjusted to $\sim 10 \mu \mathrm{g} \mathrm{ml}^{-1}$ and prepared for EM by fixing with $0.6 \%$ glutaraldehyde for $4 \mathrm{~min}$ at $20^{\circ} \mathrm{C}$, as described previously. ${ }^{18}$ The samples were observed at $40 \mathrm{kV}$ in a Tecnai $12 \mathrm{EM}$. Immuno-EM for MUC16 used CA125/ MUC16-rich material harvested from an ovarian tumor, purified on a 
$\mathrm{CsCl}$ density gradient by isopycnic centrifugation (as described above), and CA125/MUC16 taken from the material in the bottom of the gradient (fractions 18-20). After dialysis against $50 \mathrm{mM} \mathrm{Mg}$ acetate, the purified CA1 25 antigen was labeled by mixing with colloidal $10 \mathrm{~nm}$ gold pre-absorbed with the OC125 mAb. The immunogold-labeled material was isolated by HPLC on Sepharose CL2B, taking the material in the void volume, and the molecules were spread onto water, transferred to a carbon-coated grid, rotary shadowed with platinum, and examined in a JEOL 100CX scanning transmission EM (JEOL USA, Peabody, MA), as described previously. ${ }^{61}$

Atomic force microscopy. Mucins from the $\mathrm{CsCl}$ density gradient were reduced and alkylated, and dialyzed into $50 \mathrm{mM}$ ammonium hydrogen carbonate, $\mathrm{pH} 8.0$, then digested with trypsin overnight at $37^{\circ} \mathrm{C}$. The digest was chromatographed on a Sephacryl 1000 (S1000), $15 \mathrm{~cm} \times 2.5 \mathrm{~cm}$ column eluted with $200 \mathrm{mM} \mathrm{NaCl}, 10 \mathrm{mM}$ EDTA at a flow rate of $0.3 \mathrm{ml} \mathrm{min}^{-1}$. The void fraction containing the large MUC4 and MUC16 glycopeptides was collected-because the polymeric mucins were reduced and digested, their isolated, highly glycosylated mucin domains elute in the included volume of the column. ${ }^{32}$ For observation by AFM, the isolated MUC4 and MUC16 glycopeptides were mixed with $10 \mathrm{mM} \mathrm{MgCl}$, deposited on mica, air dried, and observed in an Explorer atomic force microscope (Topometrix Corp, Santa Barbara, CA) using a Nanosensors silicon probe (Neuchatel, Switzerland) at the contact mode.

SUPPLEMENTARY MATERIAL is linked to the online version of the paper at http://www.nature.com/mi

\section{ACKNOWLEDGMENTS}

We thank the Directors and teams of the UNC Cystic Fibrosis Center Tissue Culture Core, the Morphology and Morphometry Core, and the Michael Hooker Microscopy Facility for supplying reagents and technical expertise. We also thank Dr WG Matthews (Department of Physics, University of South Florida) for kindly providing the AFM images of Figure 4, Dr Surinder Batra (Department of Biochemistry and Molecular Biology, University of Nebraska Medical Center) for the kind gift of the $\lg 8 \mathrm{mAb}$, and Ms Sarah Keilson (http://sarahkeilson.com) for the illustration in Figure 8. We also thank Fujirebio Diagnostics for the kind gift of MUC1 antibodies, and Drs Jack Griffith and Sezgin Ozgur for their help in molecular EM. Valuable comments and insights into the system came from discussion with colleagues in the UNC Virtual Lung Group, Michael Rubinstein, Brian Button, and Richard Boucher, in particular. These studies were funded by grants from Cystic Fibrosis Foundation and from the National Institutes of Health (R01HL103940, R01HL77844, and P5OHL084934).

\section{DISCLOSURE}

The authors declared no conflict of interest.

() 2013 Society for Mucosal Immunology

\section{REFERENCES}

1. Knowles, M.R. \& Boucher, R.C. Mucus clearance as a primary innate defense mechanism for mammalian airways. J. Clin. Invest. 109, 571-577 (2002).

2. Fahy, J.V. \& Dickey, B.F. Airway mucus function and dysfunction. N Engl. J. Med. 363, 2233-2247 (2010).

3. Florey, H., Carleton, H.M. \& Wells, A.Q. Mucus secretion in the trachea. Br. J. Exp. Pathol. 13, 269-284 (1932).

4. Lucas, A.M. \& Douglas, L.C. Principles underlying ciliary activity in the respiratory tract. Arch. Otolaryngol. 20, 518-541 (1934).

5. Yoneda, K. Mucous blanket of rat bronchus: an ultrastructural study. Am. Rev. Respir. Dis. 114, 837-842 (1976).

6. Matsui, H. et al. Evidence for periciliary liquid layer depletion, not abnormal ion composition, in the pathogenesis of cystic fibrosis airways disease. Cell 95, 1005-1015 (1998).
7. Kesimer, M. et al. Tracheobronchial air-liquid interface cell culture: a model for innate mucosal defense of the upper airways? Am. J. Physiol. Lung Cell Mol. Physiol. 296, L92-L100 (2009).

8. Thornton, D.J., Gray, T., Nettesheim, P., Howard, M., Koo, J.S. \& Sheehan, J.K. Characterization of mucins from cultured normal human tracheobronchial epithelial cells. Am. J. Physiol. Lung Cell Mol. Physiol. 278, L1118-L1128 (2000)

9. Holmen, J.M. et al. Mucins and their O-Glycans from human bronchial epithelial cell cultures. Am. J. Physiol. Lung Cell Mol. Physiol. 287, L824-L834 (2004)

10. Ali, M., Lillehoj, E.P., Park, Y., Kyo, Y. \& Kim, K.C. Analysis of the proteome of human airway epithelial secretions. Proteome Sci. 9, 4 (2011).

11. Rahmoune, H. \& Shephard, K.L. State of airway surface liquid on guinea pig trachea. J. Appl. Physiol. 78, 2020-2024 (1995).

12. Sims, D.E. \& Horne, M.M. Heterogeneity of the composition and thickness of tracheal mucus in rats. Am. J. Physiol. 273 (5 Part 1), L1036-L1041 (1997).

13. Widdicombe, J.H. \& Widdicombe, J.G. Regulation of human airway surface liquid. Respir. Physiol. 99, 3-12 (1995).

14. Wanner, A., Salathe, M. \& O'Riordan, T.G. Mucociliary clearance in the airways. Am. J. Respir. Crit. Care Med. 154 (6 Part 1), 1868-1902 (1996).

15. Sleigh, M.A., Blake, J.R. \& Liron, N. The propulsion of mucus by cilia. Am. Rev. Respir. Dis. 137, 726-741 (1988).

16. Satir, P. \& Sleigh, M.A. The physiology of cilia and mucociliary interactions. Annu. Rev. Physiol. 52, 137-155 (1990).

17. Sheehan, J.K. \& Carlstedt, I. Hydrodynamic properties of human cervical-mucus glycoproteins in 6M-guanidinium chloride. Biochem. J. 217, 93-101 (1984).

18. Kesimer, M., Makhov, A.M., Griffith, J.D., Verdugo, P. \& Sheehan, J.K. Unpacking a gel forming mucin: a view of MUC5B organization after granular release. Am. J. Physiol. Lung Cell Mol. Physiol. 298, L15-L22 (2010).

19. Spicer, S.S., Schulte, B.A. \& Thomopoulos, G.N. Histochemical properties of the respiratory tract epithelium in different species. Am. Rev. Respir. Dis. 128 (2 Part 2), S20-S26 (1983).

20. Stonebraker, J.R. et al. Glycocalyx restricts adenoviral vector access to apical receptors expressed on respiratory epithelium in vitro and in vivo: role for tethered mucins as barriers to lumenal infection. J. Virol. 78, 13755-13768 (2004)

21. Pickles, R.J. Physical and biological barriers to viral vector-mediated delivery of genes to the airway epithelium. Proc. Am. Thorac. Soc. 1, 302-308 (2004).

22. Ebong, E.E., Macaluso, F.P., Spray, D.C. \& Tarbell, J.M. Imaging the endothelial glycocalyx in vitro by rapid freezing/freeze substitution transmission electron microscopy. Arterioscler. Thromb. Vasc. Biol. 31 1908-1915 (2011)

23. Rose, M.C. \& Voynow, J.A. Respiratory tract mucin genes and mucin glycoproteins in health and disease. Physiol. Rev. 86, 245-278 (2006).

24. Thornton, D.J., Rousseau, K. \& McGuckin, M.A. Structure and function of the polymeric mucins in airways mucus. Annu. Rev. Physiol. 70, 459-486 (2008).

25. Gouyer, V., Gottrand, F. \& Desseyn, J.L. The extraordinarily complex but highly structured organization of intestinal mucus-gel unveiled in multicolor images. PLOS ONE 6, e18761 (2011).

26. Aplin, J.D. MUC-1 glycosylation in endometrium: possible roles of the apical glycocalyx at implantation. Hum. Reprod. 14 (Suppl 2), 17-25 (1999).

27. Zhang, L. et al. Infection of ciliated cells by human parainfluenza virus type 3 in an in vitro model of human airway epithelium. J. Virol. 79, 1113-1124 (2005).

28. Monzon, M.E., Casalino-Matsuda, S.M. \& Forteza, R.M. Identification of glycosaminoglycans in human airway secretions. Am. J. Respir. Cell Mol. Biol. 34, 135-141 (2006).

29. Kwilas, S., Liesman, R.M., Zhang, L., Walsh, E., Pickles, R.J. \& Peeples, M.E. Respiratory syncytial virus grown in Vero cells contains a truncated attachment protein that alters its infectivity and dependence on glycosaminoglycans. J. Virol. 83, 10710-10718 (2009).

30. Jeffery, P.K. Structure and function of mucus-secreting cells of cat and goose airway epithelium. Ciba. Found Symp. 54, 5-23 (1978).

31. Carlstedt, I., Lindgren, H., Sheehan, J.K., Ulmsten, U. \& Wingerup, L. Isolation and characterization of human cervical-mucus glycoproteins. Biochem. J. 211, 13-22 (1983).

32. Carlstedt, I., Lindgren, H. \& Sheehan, J.K. The macromolecular structure of human cervical-mucus glycoproteins. Studies on fragments obtained 
after reduction of disulphide bridges and after subsequent trypsin digestion. Biochem. J. 213, 427-435 (1983).

33. Higuchi, T. et al. Molecular cloning, genomic structure, and expression analysis of MUC20, a novel mucin protein, up-regulated in injured kidney. J. Biol. Chem. 279, 1968-1979 (2004).

34. Finkbeiner, W.E., Zlock, L.T., Morikawa, M., Lao, A.Y., Dasari, V. \& Widdicombe, J.H. Cystic fibrosis and the relationship between mucin and chloride secretion by cultures of human airway gland mucous cells. Am. J. Physiol. Lung Cell Mol. Physiol. 301, L402-L414 (2011).

35. Cao, R., Wang, T., Demaria, G., Sheehan, J. \& Kesimer, M. Mapping the protein domain structures of the respiratory mucins: a mucin proteome coverage study. J. Proteome. Res. 11, 4013-4023 (2012).

36. Madsen, J., Mollenhauer, J. \& Holmskov, U. Gp-340/DMBT1 in mucosal innate immunity. Innate Immun. 16, 160-167 (2010).

37. Yin, B.W., Dnistrian, A. \& Lloyd, K.O. Ovarian cancer antigen CA125 is encoded by the MUC16 mucin gene. Int. J. Cancer 98, 737-740 (2002).

38. Maeda, T. et al. Solution structure of the SEA domain from the murine homologue of ovarian cancer antigen CA125 (MUC16). J. Biol. Chem. 279, 13174-13182 (2004).

39. Hattrup, C.L. \& Gendler, S.J. Structure and function of the cell surface (tethered) mucins. Annu. Rev. Physiol. 70, 431-457 (2008).

40. Carrion-Vazquez, M. et al. Mechanical and chemical unfolding of a single protein: a comparison. Proc. Natl. Acad. Sci. USA 96, 3694-3699 (1999).

41. Van den Steen, P., Rudd, P.M., Dwek, R.A. \& Opdenakker, G. Concepts and principles of O-linked glycosylation. Crit. Rev. Biochem. Mol. Biol. 33, 151-208 (1998).

42. Burke, P.A. et al. Characterization of MUC1 glycoprotein on prostate cancer for selection of targeting molecules. Int. J. Oncol. 29, 49-55 (2006).

43. Kesimer, M. et al. Characterization of exosome-like vesicles released from human tracheobronchial ciliated epithelium: a possible role in innate defense. FASEB J. 23, 1858-1868 (2009).

44. Nustad, K. et al. Epitopes on CA 125 from cervical mucus and ascites fluid and characterization of six new antibodies. Third report from the ISOBM TD-1 workshop. Tumour Biol. 23, 303-314 (2002).

45. McDowell, E.M., Barrett, L.A., Glavin, F., Harris, C.C. \& Trump, B.F. The respiratory epithelium. I. Human bronchus. J. Natl. Cancer Inst. 61 , 539-549 (1978).

46. Lopez-Vidriero, M.T. Mucus as a natural barrier. Respiration 55 (Suppl 1), 28-32 (1989)

47. Olmsted, S.S., Padgett, J.L., Yudin, A.I., Whaley, K.J., Moench, T.R. \& Cone, R.A. Diffusion of macromolecules and virus-like particles in human cervical mucus. Biophys. J. 81, 1930-1937 (2001).

48. Sears, P.R., Davis, C.W., Chua, M. \& Sheehan, J.K. Mucociliary interactions and mucus dynamics in ciliated human bronchial epithelial cell cultures. Am. J. Physiol. Lung Cell Mol. Physiol. 301, L181-L186 (2011).

49. Davies, J.R., Kirkham, S., Svitacheva, N., Thornton, D.J. \& Carlstedt, I. MUC16 is produced in tracheal surface epithelium and submucosal glands and is present in secretions from normal human airway and cultured bronchial epithelial cells. Int. J. Biochem. Cell Biol. 39, 1943-1954 (2007)

50. de Mesy Bentley, K.L. An 11-mum-thick glycocalyx? it's all in the technique!. Arterioscler. Thromb. Vasc. Biol. 31, 1712-1713 (2011).
51. Button, B. et al. A periciliary brush promotes lung health by separating the mucus layer from airway epithelia. Science, doi:10.1126/ science.1223012 (in press) (2012)

52. Kesimer, M. \& Sheehan, J.K. Analyzing the functions of large glycoconjugates through the dissipative properties of their absorbed layers using the gel-forming mucin MUC5B as an example. Glycobiology 18, 463-472 (2008).

53. Desseyn, J.L., Tetaert, D. \& Gouyer, V. Architecture of the large membrane-bound mucins. Gene 410, 215-222 (2008).

54. Williams, S.J., Wreschner, D.H., Tran, M., Eyre, H.J., Sutherland, G.R. \& McGuckin, M.A. Muc13, a novel human cell surface mucin expressed by epithelial and hemopoietic cells. J. Biol. Chem. 276, 18327-18336 (2001).

55. Pallesen, L.T., Pedersen, L.R., Petersen, T.E., Knudsen, C.R. \& Rasmussen, J.T. Characterization of human mucin (MUC15) and identification of ovine and caprine orthologs. J. Dairy Sci. 91, 4477-4483 (2008).

56. Harden, J.L. \& Cates, M.E. Extension and compression of grafted polymer layers in strong normal flows. J. Physique // 5, 1093-1103 (1995).

57. Raviv, U., Giasson, S., Kampf, N., Gohy, J.F., Jerome, R. \& Klein, J. Lubrication by charged polymers. Nature 425, 163-165 (2003).

58. Fulcher, M.L., Gabriel, S., Burns, K.A., Yankaskas, J.R. \& Randell, S.H. Well-differentiated human airway epithelial cell cultures. Methods Mol. Med. 107, 183-206 (2005).

59. Pickles, R.J., McCarty, D., Matsui, H., Hart, P.J., Randell, S.H. \& Boucher, R.C. Limited entry of adenovirus vectors into well-differentiated airway epithelium is responsible for inefficient gene transfer. J. Virol. 72, 6014-6023 (1998).

60. Thornton, D.J., Howard, M., Devine, P.L. \& Sheehan, J.K. Methods for separation and deglycosylation of mucin subunits. Anal. Biochem. 227, 162-167 (1995).

61. Sheehan, J.K., Oates, K. \& Carlstedt, I. Electron microscopy of cervical, gastric and bronchial mucus glycoproteins. Biochem. J. 239, 147-153 (1986).

62. Grinstead, J.S., Schuman, J.T. \& Campbell, A.P. Epitope mapping of antigenic MUC1 peptides to breast cancer antibody fragment B27.29: a heteronuclear NMR study. Biochemistry 42, 14293-14305 (2003).

63. Wesseling, J., van der Valk, S.W., Vos, H.L., Sonnenberg, A. \& Hilkens, J. Episialin (MUC1) overexpression inhibits integrin-mediated cell adhesion to extracellular matrix components. J. Cell Biol. 129, 255-265 (1995).

64. Hovenberg, H.W., Davies, J.R., Herrmann, A., Linden, C.J. \& Carlstedt, I. MUC5AC, but not MUC2, is a prominent mucin in respiratory secretions. Glycoconj. J. 13, 839-847 (1996).

65. Lidell, M.E., Bara, J. \& Hansson, G.C. Mapping of the 45M1 epitope to the $\mathrm{C}$-terminal cysteine-rich part of the human MUC5AC mucin. FEBS J. 275, 481-489 (2008).

66. Rousseau, K., Wickstrom, C., Whitehouse, D.B., Carlstedt, I. \& Swallow, D.M. New monoclonal antibodies to non-glycosylated domains of the secreted mucins MUC5B and MUC7. Hybrid Hybridomics 22, 293-299 (2003).

67. Young, R.D., Gealy, E.C., Liles, M., Caterson, B., Ralphs, J.R. \& Quantock, A.J. Keratan sulfate glycosaminoglycan and the association with collagen fibrils in rudimentary lamellae in the developing avian cornea. Invest. Ophthalmol. Vis. Sci. 48, 3083-3088 (2007). 